\title{
Modified Biweekly Oxaliplatin and Capecitabine for Advanced Gastric Cancer: A Retrospective Analysis from A Medical Center
}

\author{
Yung-Chia Kuo ${ }^{1}$, Hao-Tien Liu ${ }^{2}$, Yi-Lun Lin ${ }^{2}$, Yi-Chun Yang ${ }^{2}$, Tsai-Sheng Yang ${ }^{1}$, Chi-Ting Liau ${ }^{1}$, \\ Wen-Chi Shen ${ }^{1}$, Hung-Chih Hsu' ${ }^{1}$, Wen-Chi Chou ${ }^{1}$, Jen-Shi Chen ${ }^{1,2}$
}

Background: We modified 3-week XELOX regimen with oxaliplatin to $85 \mathrm{mg} / \mathrm{m}^{2}$ on Day 1 and capecitabine $1000 \mathrm{mg} / \mathrm{m}^{2}$ BID for 10 days every 14 days to be more practical in clinical practice for advanced gastric cancer. The aim of this retrospective analysis is to evaluate the safety profile and efficacy of the modified oxaliplatin plus capecitabine (XELOX) regimen as the first-line treatment for patients with advanced gastric cancer in a medical center in Taiwan.

Methods: $\quad$ From March 2009 to December 2010, among the 614 patients diagnosed with gastric cancer in a medical center, 49 patients with unresectable advanced or metastatic gastric adenocarcinoma were treated with oxaliplatin $\left(85 \mathrm{mg} / \mathrm{m}^{2}\right)$ on Day 1 and capecitabine (1000 mg/m² BID) for 10 days every 2 weeks (mXELOX). CT scan was performed for tumor response evaluation. Clinical outcome and adverse events after mXELOX treatment were analyzed retrospectively.

Results: A total of 354 mXELOX sessions (median: 6) were administered in 49 patients. The overall tumor response rate was $39.1 \%$ among 46 evaluated patients: three complete response (6.5\%) and 15 partial response (32.6\%). Seven patients had stable disease (15.2\%) and 21 (45.7\%) patients had progressive disease. The median progression-free survival and median overall survival were 4.37 months and 12.26 months, respectively. The most common grade III/IV hematologic toxicity was anemia $(10.2 \%)$, and non-hematologic toxicity effects were numbness $(8.2 \%)$, hand-foot syndrome (10.2\%), diarrhea (6.1\%), thrombocytopenia (6.1\%), and abdominal pain $(6.1 \%)$.

Conclusion: This modified biweekly oxaliplatin and capecitabine combination chemotherapy is practical and effective for unresectable advanced or metastatic gastric cancer in our daily practice.

(Biomed J 2014;37:141-146)

\begin{abstract}
At a Glance Commentary
Scientific background of the subject

Capecitabine and oxaliplatin are widely used in the treatment of advanced gastric cancer based on previous studies and reimbursed by the National Health Insurance of Taiwan since 2009. The toxicities of the conventional triweekly XELOX caused poor compliance and led to dose modifications, dose reductions, cycle interruptions, and cycle delays.
\end{abstract}

\section{What this study adds to the field}

Compared with the conventional XELOX, this study as a retrospective analysis of modified biweekly XELOX for advanced gastric cancer showed acceptable safety profile, and similar response rate under similar dose intensity. This result reveals that this regimen is more practical with less acute toxicities, and is similarly effective in our daily practice.

\section{Key words: advanced gastric cancer, capecitabine, oxaliplatin}

G astric cancer (GC) is one of the most common malignant cancers of the world ${ }^{[1]}$ and is the $7^{\text {th }}$ most common cancer in Taiwan. ${ }^{[2,3]}$ Till date, surgical resection is the only curative therapy for localized GC. For patients with unresectable GC, palliative chemotherapy has demonstrated benefits of improving the quality of life and prolonging survival time

From the ${ }^{1}$ Division of Hematology/Oncology, Department of Internal Medicine, Chang Gung Memorial Hospital at Linkou, Chang Gung University College of Medicine, Taoyuan, Taiwan; ${ }^{2}$ School of Medicine, College of Medicine, Chang Gung University, Taoyuan, Taiwan Received: Feb. 18, 2013; Accepted: Jun. 19, 2013

Correspondence to: Dr. Jen-Shi Chen, Division of Hematology/Oncology, Department of Internal Medicine, Chang Gung Memorial Hospital at Linkou. 5, Fuhsing St. Guieshan, Taoyuan, Taiwan. Tel: 886-3-3281200 ext. 8825; Fax: 886-3-3278211;

E-mail: js1101@adm.cgmh.org.tw

DOI: $10.4103 / 2319-4170.117887$ 
than the best supportive care. ${ }^{[4,5]}$ Previous studies, ${ }^{[4,6]}$ show that combination chemotherapy is superior than single-agent chemotherapy with respect to overall survival (OS), tumor response, and progression-free survival (PFS).

The widely used combination chemotherapy nowadays is with fluoropyridines ([fluorouracil (5-FU)]-based and platinum-based regimens. ${ }^{[4,6]}$ Miwa et al.,${ }^{[7]}$ have demonstrated capecitabine, the oral form pro-drug of 5-FU, can achieve better tumor concentration/localization of 5-FU than parenteral 5-FU in human liver and cancer tissue. Capecitabine with cisplatin or oxaliplatin combinations have already shown the same benefits in OS and response rate compared with 5-FU and cisplatin or oxaliplatin combinations in advanced GC. ${ }^{[8]}$ In addition, meta-analysis for capecitabine-based combination has shown it to be more effective than 5-FU infusion in GC. Moreover, the advantages of capecitabine over continuous 5-FU infusion include the convenience of oral chemotherapy, which is generally more acceptable to patients, and avoiding the potential morbidity associated with central venous access. ${ }^{[8]}$ Oxaliplatin is an alkylating agent of third-generation platinum which inhibits DNA replication. Compared with cisplatin, oxaliplatin appears to have a more favorable safety profile and less cross-resistance to cisplatin. ${ }^{\left[{ }^{-12]}\right.}$ Keeping the efficacy, adverse effects, and convenience into consideration, it is reasonable to use capecitabine and oxaliplatin instead of 5-FU and cisplatin.

Based on the above evidences, the National Health Insurance of Taiwan has reimbursed combination of capecitabine and oxaliplatin for advanced GC since 2009. The response rates of conventional combination regimen of capecitabine and oxaliplatin (XELOX) for GC ranged between $34 \%$ and $65 \% .{ }^{[13-19]}$ XELOX generally consisted of the oxaliplatin $130 \mathrm{mg} / \mathrm{m}^{2}$ on Day 1 and capecitabine (XELODA $^{\circledR}$, Roche, Switzerland) $1000 \mathrm{mg} / \mathrm{m}^{2}$ BID for 14 days every 3 weeks. ${ }^{[13-19]}$ The most common adverse events of triweekly XELOX were reported by The capecitabine and oxaliplatin adjuvant study in stomach cancer (CLASSIC) study, which enrolled more than 500 cases of GC for adjuvant treatment. The major adverse events included nausea $(66 \%)$, neutropenia $(60 \%)$, peripheral neuropathy $(56 \%)$, diarrhea (48\%), vomiting (39\%), thrombocytopenia (26\%), and unique hand-foot syndrome (HFS, 19\%) in this recent large-scale study. ${ }^{[13]}$ These adverse events caused poor compliance of patients. In this trial, only $67 \%$ of patients completed planned cycles. Adverse events led to chemotherapy dose modifications in $446(90 \%)$ patients. Thirty-four percent of patients had capecitabine dose reductions, $30 \%$ had cycle interruptions, $74 \%$ had cycle delays, and $33 \%$ of patients needed oxaliplatin dose reductions. ${ }^{[13]}$

An alternative dose-dense regimen of oxaliplatin at $85 \mathrm{mg} / \mathrm{m}^{2}$ on Day 1 and capecitabine at $2500-4000 \mathrm{mg} / \mathrm{m}^{2} /$ day on Day 1-7 every 2 weeks (Q2W) was developed and found to be active with an acceptable safety profile in advanced colorectal cancer ${ }^{[14]}$ Compared to triweekly capecitabine $\left(850 \mathrm{mg} / \mathrm{m}^{2}, \mathrm{BID}\right.$, Day 1-14), dose-dense biweekly capecitabine $\left(1500 \mathrm{mg} / \mathrm{m}^{2}\right.$, BID, Day 1-7) had higher incidences for diarrhea, nausea, vomiting, and HFS. ${ }^{[15]}$ Consequently, it is reasonable to modify triweekly XELOX with oxaliplatin to $85 \mathrm{mg} / \mathrm{m}^{2}$ on Day 1 and capecitabine to $1000 \mathrm{mg} / \mathrm{m}^{2}$ BID for 10 days every 14 days to be more practical and tolerable in clinical practice.

This retrospective analysis was carried out with an aim to evaluate the safety profile and efficacy of modified XELOX regimen as the first-line treatment for patients with adfvanced GC in Taiwan.

\section{METHODS}

\section{Patients}

From February 2009 to December 2010, there were 614 patients who had histological or cytological diagnosis of GC in Chang-Gung Memorial Hospital. Among these patients, we reviewed 49 patients who had inoperable locally advanced primary or metastatic gastric adenocarcinoma treated with modified XELOX regimen as first-line palliative chemotherapy by physician' discretion.

\section{Treatment schedule}

The modified XELOX regimen was consisted of capecitabine (1000 mg/m² twice daily, Day 1-10) plus oxaliplatin $\left(85 \mathrm{mg} / \mathrm{m}^{2}\right.$ as a2-h intravenous infusion on Day 1) every 2 weeks. For intolerance to oxaliplatin manifested as neurotoxicity with numbness, capecitabine alone was continued while oxaliplatin was discontinued. Treatment was continued until disease progression, intolerable adverse effects, patient's refusal, or physician's discretion.

\section{Evaluation of response and toxicity}

The patients underwent physical examination, complete blood counts, and serum biochemistry analyses before the first treatment cycle. Following complete blood cell counts with differential counts, serum biochemistry analyses were performed every 2 weeks before each session. Response was evaluated by physical examination, chest plain films, and series of abdominal-pelvic CT scan. Response was assessed according to RECIST criteria version 1.0. Assessment of PFS was determined by measuring the time interval from the beginning of treatment until the first documented progression or patient death. OS was defined as the period from the beginning of treatment to the day of death from any cause. Adverse events were evaluated according to the grading system of National Cancer Institute, Common Terminology Criteria for Adverse Events (NCI CTCAE) Version 3.0. 


\section{Statistical analyses}

The primary endpoints of this analysis were overall response rate and the adverse events of this regimen. The secondary endpoints of this study were PFS and OS. The response rate was analyzed with intent-to-treat. PFS and OS were analyzed by Kaplan-Meier method. All data were analyzed by SPSS software (SPSS 19.0 Inc., Chicago, IL, USA).

\section{RESULTS}

From February 2009 to December 2010, 49 patients consecutively treated with mXELOX regimen were enrolled for this analysis. There were 28 males and 21 females with median age of 59 years (range 28-78 years). Nine patients received surgical treatment prior to $\mathrm{mXELOX}$ treatment. Seven of them were treated as metastatic disease under clinical judgments, while two patients with metastatic disease were found during operation. Baseline patient characteristics are listed in Table 1. There were 354 total treatment sessions with a median session of 6 (range 1-19) in 49 patients. Ten patients (20.4\%) received capecitabine alone with a median session of 7.5 (range 1-28) after ceasing oxaliplatin. The reasons for ceasing this treatment were adverse events in 22 patients ( $44 \%$ ), disease progression in 19 patients (38\%), loss of follow-up in 4 patients (8\%), and complete response (CR) in 4 patients [Table 2].

There were 49 patients enrolled to assess toxicity in this study. No grade 4 toxicity was noted. In all grades of adverse events [Table 3], the most common hematological toxicity was anemia and the most common non-hematological toxicity was numbness. The most common grade 3 toxicity was anemia $[5 / 49(10.2 \%)]$, which was followed by numbness [4/49 (8.2\%)], HFS [5/49 (10.2\%)], diarrhea $[3 / 49(6.1 \%)]$, thrombocytopenia [3/49 $(6.1 \%)]$, and abdominal pain [3/49 (6.1\%)] [Table 3]. However, among these patients, there were three patient deaths during treatment. One died of sepsis with undetermined focus, another died of pneumonia, and the cause of the other death was biliary tract infection. None of these patients had neutropenia or other chemotherapy-related adverse events.

There were 46 patients for response evaluation. The overall response rate was $39.1 \%$, including 3 patients with $\mathrm{CR}(6.5 \%)$ and 15 patients with partial response (PR, 32.6\%). Seven patients had stable disease (SD, 15.2\%) and 21 patients had progressive disease [Table 4]. The disease-control rate $(\mathrm{CR}+\mathrm{PR}+\mathrm{SD})$ was $54.3 \%$. The median PFS was 4.37 months (95\% CI 1.88-6.86 months) [Figure 1] and median OS was 12.26 months (95\% CI 9.59-14.92 months) [Figure 2].

\section{DISCUSSION}

The modified XELOX regimen used in this study demonstrated quite an acceptable response rate and survival

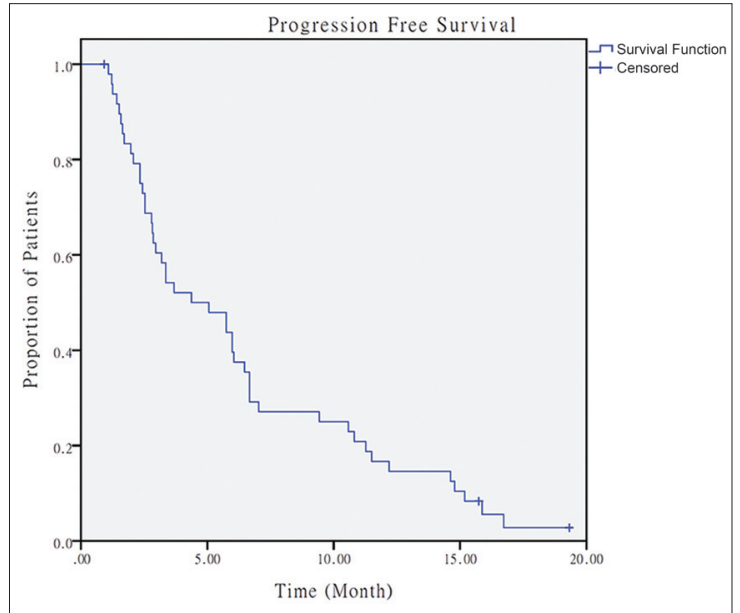

Figure 1: Progression-free survival

Table 1: Patient characteristics, $n=49$

\begin{tabular}{lc}
\hline Characteristics & Number of patients (\%) \\
\hline Age, median & 59 \\
$\quad$ Range & $28-77$ \\
Gender & \\
Male & $28(57)$ \\
Female & $21(43)$ \\
Performance status (ECOG) & \\
0 & $21(42.9)$ \\
1 & $24(49.0)$ \\
$\geq 2$ & $4(8.1)$ \\
Stage & \\
2 & $3(6.1)$ \\
3 & $6(12.2)$ \\
4 & $40(81.7)$ \\
Gastrectomy or bypass surgery & \\
No & 40 \\
Yes & 9 \\
Evaluated for response & 46 \\
Not assessed & 3
\end{tabular}

Abbreviation: ECOG: Eastern cooperative oncology group

Table 2: The reasons for off-treatment of mXELOX regimen

\begin{tabular}{lc}
\hline & No. of patients (\%) \\
\hline Toxicity & $22(44)$ \\
Neuropathy & $7(14)$ \\
GI symptoms & $5(10)$ \\
Leukopenia & $1(2)$ \\
Thrombocytopenia & $2(4)$ \\
Hand foot syndrome & $2(4)$ \\
Infection (including mortality*) & $4(8)^{*}$ \\
Allergy & $1(2)$ \\
Disease progression & $19(34)$ \\
Physician decision & $4(8)$ \\
Transfer or loss to follow-up/patient refusal & $4(8)$ \\
*Three patients died of infection (6\%), all were cancer related, none related
\end{tabular}

to bone marrow toxicity. Abbreviation: mXELOX: modified XELOX 


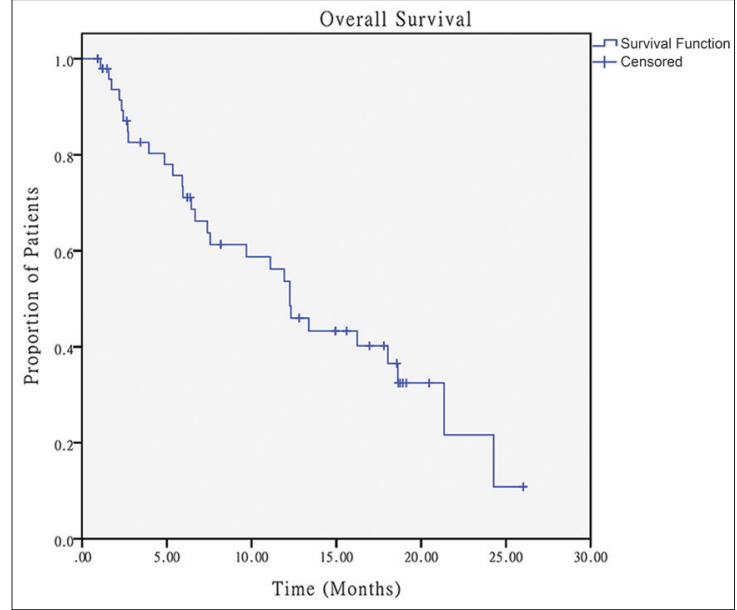

Figure 2: Overall survival

time. There is similar dose intensity between conventional XELOX regimen and modified XELOX regimen. An important feature of mXELOX in this study is the shorter period of administration of capecitabine. Compared with the results of conventional XELOX regimens [Table 5], the major grade $3 / 4$ adverse effects of modified XELOX were around $10 \%$. The incidences of neutropenia, leucopenia, and thrombocytopenia were lower in $\mathrm{mXELOX.} \mathrm{The} \mathrm{number} \mathrm{of}$ deaths was three during the treatment period, but none of them died of treatment-related adverse events. Furthermore, gastrointestinal (GI) adverse events (nausea, vomiting, and diarrhea) of this series were less than those of Park et al. ${ }^{[16,22]}$ and other reports. It implies that less capecitabine-related hematological and GI adverse events occurred under shorter period of administration of capecitabine at similar dose intensity.

Meanwhile, the most common associated hematologic event related to this regimen was anemia [42/49, (85.7\%)]. Grade 3/4 anemia was $10.2 \%$, higher than that reported by Park et al., ${ }^{[16,20]}$ and in other studies. This difference could be explained by the underlying anemia that was suspected to be associated with patients who had previously undergone total or subtotal gastrectomy, which we did not exclude. As capecitabine often causes HFS, the overall incidence in our study was less than in previous phase II trials. But it is similar to CLASSIC phase III trial. Grade 3 HFS was about $10 \%$, similar to that reported by Dong et al ${ }^{[17]}$ It is believed that capecitabine is more tolerable in Asians and Europeans than in US patients. The reasons for this discrepancy between Europeans and US patients are not known, but it is believed to be related to the US diet fortified with folic acid and folic acid exacerbates capecitabine toxicity, compliance, or some genetic variations. ${ }^{[23]}$ To our surprise, HFS in Asians remained an important adverse event. Further studies are necessary to clarify the toxicity profile of capecitabine among different ethnic groups.
Table 3: Adverse events of $\mathrm{mXELOX}, n=49$

\begin{tabular}{lcl}
\hline & \multicolumn{2}{c}{ No. of patients $(\%)$} \\
\cline { 2 - 3 } & Grade 1 and 2 & Grade $3 *$ \\
\hline Hematologic toxicity & $37(75.5)$ & $5(10.2)$ \\
Anemia & $11(22.5)$ & $1(2.0)$ \\
Leucopenia & $8(16.3)$ & $1(2.0)$ \\
Neutropenia & $15(30.6)$ & $3(6.12)$ \\
Thrombocytopenia & $0(0)$ & $1(2.0)$ \\
Infection & & \\
Non-hematologic toxicity & $12(24.5)$ & $1(2.0)$ \\
Renal insufficiency & $5(10.2)$ & $0(0)$ \\
Mucositis & $18(36.7)$ & $2(4.1)$ \\
Anorexia & $13(26.5)$ & $1(2.0)$ \\
Nausea/vomiting & $12(24.5)$ & $3(6.1)$ \\
Diarrhea & $6(12.2)$ & $3(6.1)$ \\
Abdominal pain & $14(28.6)$ & $1(2.0)$ \\
Fatigue & $18(36.7)$ & $4(8.2)$ \\
Neuropathy & $6(12.2)$ & $5(10.2)$ \\
Hand-foot syndrome &
\end{tabular}

*No grade 4 toxicity was observed. Abbreviations: mXELOX: modified XELOX

Table 4: Efficacy and survival of $m X E L O X$ regime, $n=46$

\begin{tabular}{lc}
\hline & No. of patients $(\%)$ \\
\hline Response rate & $18(39.13)$ \\
Complete response & $3(6.52)$ \\
Partial response & $15(32.61)$ \\
Stable disease & $7(15.22)$ \\
Progressive disease & $21(45.65)$
\end{tabular}

Median time to disease progression $\quad 4.35$ months (95\% CI 1.54-7.16)

Median overall survival $\quad 12.03$ months (95\% CI 9.68-14.38)

Abbreviations: mXELOX: modified XELOX; CI: Confidence interval

Table 5: Literatures for XELOX in AGC, toxicity: Grade $1-2 / 3-4(\%)$

\begin{tabular}{|c|c|c|c|c|c|}
\hline & \multicolumn{5}{|c|}{ References } \\
\hline & $\begin{array}{l}\text { Jatoi }^{[20]} \\
(2006)\end{array}$ & $\begin{array}{c}\mathrm{Liu}^{[21]} \\
(2008)\end{array}$ & $\begin{array}{l}\operatorname{Park}^{[16]} \\
(2008)\end{array}$ & $\begin{array}{l}\text { Dong }{ }^{[17]} \\
(2009)\end{array}$ & $\begin{array}{l}\text { This } \\
\text { study }\end{array}$ \\
\hline Anemia & NA* & $69.3 / 0$ & $70 / 0$ & $54.5 / 2.3$ & $75.5 / 10$ \\
\hline Leukopenia & NA & $41.6 / 4.6$ & $42 / 6$ & $40.9 / 6.8$ & $22.5 / 2$ \\
\hline Neutropenia & NA & $38.5 / 6.1$ & $40 / 8$ & $36.4 / 13.6$ & $16.3 / 2$ \\
\hline Thrombocytopenia & NA & $21.5 / 7.7$ & $24 / 11$ & $27.3 / 11.3$ & $30.6 / 6$ \\
\hline Nausea/Vomiting & 46 & $50.8 / 1.5$ & $50 / 2$ & $43.2 / 4.5$ & $44.9 / 4$ \\
\hline Diarrhea & 30 & $26.2 / 6.2$ & $26 / 7$ & $52.2 / 13.6$ & $24.5 / 2$ \\
\hline HFS & NA & $40 / 0$ & $39 / 0$ & $40.9 / 9.1$ & $12.2 / 10$ \\
\hline Anorexia & 11 & NA & NA & NA & $36.7 / 4.1$ \\
\hline Neuropathy & NA & $67.7 / 0$ & $70 / 0$ & $43.1 / 0$ & $36.7 / 8.2$ \\
\hline
\end{tabular}

*Abbreviations: NA: Not-available; HFS: Hand-foot syndrome; AGC: Advanced gastric cancer

Although the incidence of neutropenia and anemia was less, there were four patients who encountered grade 3 neuropathy while seven patients discontinued from mXELOX regimen due to neuropathy and physician decision. It represents that oxaliplatin still causes cumulative sensory pe- 
ripheral neuropathy, which limits the dose of oxaliplatin, ${ }^{[1]}$ and patient tolerance may be individualized. The incidence of neurotoxicity, the most common adverse effect in our study, leading patients to cease chemotherapy, was different from that reported by Park et al. ${ }^{[16,22]}$ but similar to the report by Bang et al.$^{[13]}$ In our data, the median of oxaliplatin administration was six sessions, which was ceased due to neurotoxicity. In addition, the incidences of neuropathy of all grades were similar, but more grade 3 neuropathy was noted with mXELOX compared with the CLASSIC trial. According to the report of Park et al., report, severe neurotoxicity of oxaliplatin is strongly cumulative dose dependent and results in significant limitation to treatment. ${ }^{[19]}$ It may cause our treatment duration shorter than the conventional triweekly XELOX, while similar dose intensity was achieved earlier.

Although our patients received shorted treatment duration under similar dose intensity, in this retrospective analysis, the overall response rate of modified XELOX regimen was $39.14 \%$. In addition, the median PFS was 4.37 months and median OS was 12.26 months. It shows the result of our daily practice was compatible with those of previous phase II studies (the overall response rate was $34-65 \%$, PFS was 3.2-7.5 months, and OS was 6.4-11.9 months), in which the results can provide a strong evidence to support National Health Insurance of Taiwan to reimburse XELOX regimens for advanced GC.

Since the dose intensity in this study is similar to that in other previous studies, less side effects without compromised dose intensity may improve treatment compliance. Under comparable response rates and similar treatment doses among these studies, this mXELOX regimen had less acute toxicities of capecitabine, but did not decrease chronic or long-term toxicities of oxaliplatin. Our data showed that mXELOX regimen was effective and tolerable as the first-line palliative chemotherapy for advanced GC. However, these results should be confirmed by prospective phase III studies, and further analyses for uni-variant and multi-variants to verify the prognosis factor should be conducted with large-scale and multiple institutes.

\section{Conclusion}

The modified biweekly XELOX combination chemotherapy is practical and effective for unresectable advanced or metastatic GC in daily practice.

\section{REFERENCES}

1. Hartgrink HH, Jansen EP, van Grieken NC, van de Velde CJ. Gastric cancer. Lancet 2009;374:477-90

2. Chiang CJ, Chen YC, Chen CJ, You SL, Lai MS; Taiwan Cancer Registry Task Force. Cancer Trends in Taiwan. Jpn J Clin Oncol 2010;40:897-904.
3. Bureau of Health Promotion DoH, The Executive Yuan, Taiwan, R.O.C. Cancer Registry Annual Report, 2009 Taiwan. 2012.

4. Wagner AD, Grothe W, Haerting J, Kleber G, Grothey A, Fleig WE. Chemotherapy in advanced gastric cancer: A systematic review and meta-analysis based on aggregate data. J Clin Oncol 2006;24:2903-9.

5. Rivera F, Vega-Villegas ME, López-Brea MF. Chemotherapy of advanced gastric cancer. Cancer Treat Rev 2007;33:315-24.

6. Wöhrer SS, Raderer M, Hejna M. Palliative chemotherapy for advanced gastric cancer. Ann Oncol 2004;15:1585-95.

7. Miwa M, Ura M, Nishida M, Sawada N, Ishikawa T, Mori K, et al. Design of a novel oral fluoropyrimidine carbamate, capecitabine, which generates 5-fluorouracil selectively in tumours by enzymes concentrated in human liver and cancer tissue. Eur J Cancer 1998;34:1274-81.

8. Okines AF, Norman AR, McCloud P, Kang YK, Cunningham D. Meta-analysis of the REAL-2 and ML17032 trials: Evaluating capecitabine-based combination chemotherapy and infused 5-fluorouracil-based combination chemotherapy for the treatment of advanced oesophago-gastric cancer. Ann Oncol 2009;20:1529-34.

9. Cunningham D, Starling N, Rao S, Iveson T, Nicolson M, Coxon F, et al. Capecitabine and oxaliplatin for advanced esophagogastric cancer. N Engl J Med 2008;358:36-46.

10. Kang YK, Kang WK, Shin DB, Chen J, Xiong J, Wang J, et al. Capecitabine/cisplatin versus 5-fluorouracil/cisplatin as first-line therapy in patients with advanced gastric cancer: A randomised phase III noninferiority trial. Ann Oncol 2009;20:666-73.

11. Montagnani F, Turrisi G, Marinozzi C, Aliberti C, Fiorentini G. Effectiveness and safety of oxaliplatin compared to cisplatin for advanced, unresectable gastric cancer: A systematic review and meta-analysis. Gastric Cancer 2011;14:50-5.

12. Wong R, Cunningham D. Optimising treatment regimens for the management of advanced gastric cancer. Ann Oncol 2009;20:605-8.

13. Bang YJ, Kim YW, Yang HK, Chung HC, Park YK, Lee KH, et al.; CLASSIC trial investigators. Adjuvant capecitabine and oxaliplatin for gastric cancer after D2 gastrectomy (CLASSIC): A phase 3 open-label, randomised controlled trial. Lancet Oncol 2012;379:315-21.

14. Scheithauer W, Kornek GV, Raderer M, Schüll B, Schmid K, Längle F, et al. Intermittent weekly high-dose capecitabine in combination with oxaliplatin: A phase I/II study in first-line treatment of patients with advanced colorectal cancer. Ann Oncol 2002;13:1583-9.

15. Hurwitz H, Mitchell EP, Cartwright T, Kwok A, Hu S, McKenna E, et al. A Randomized, phase ii trial of standard triweekly compared with dose-dense biweekly capecitabine plus oxaliplatin plus bevacizumab as first-line treatment for metastatic colorectal cancer: XELOX-A-DVS (Dense Versus Standard). Oncologist 2012;17:937-46.

16. Park YH, Lee JL, Ryoo BY, Ryu MH, Yang SH, Kim BS, et al. Capecitabine in combination with Oxaliplatin (XELOX) as a first-line therapy for advanced gastric cancer. Cancer Chemother Pharmacol 2008;61:623-9.

17. Dong N, Jiang W, Li H, Liu Z, Xu X, Wang M. Triweekly oxaliplatin plus oral capecitabine as first-line chemotherapy in elderly patients with advanced gastric cancer. Am J Clin Oncol 2009;32:559-63.

18. Saif MW, Choma A, Salamone SJ, Chu E. Pharmacokinetically guided dose adjustment of 5-fluorouracil: A rational approach to improving therapeutic outcomes. J Natl Cancer Inst 2009;101:1543-52. 
19. Park SB, Lin CS, Krishnan AV, Goldstein D, Friedlander ML, Kiernan MC. Oxaliplatin-induced neurotoxicity: Changes in axonal excitability precede development of neuropathy. Brain 2009;132:2712-23.

20. Jatoi A, Murphy BR, Foster NR, Nikcevich DA, Alberts SR, Knost JA, et al. Oxaliplatin and capecitabine in patients with metastatic adenocarcinoma of the esophagus, gastroesophageal junction and gastric cardia: A phase II study from the North Central Cancer Treatment Group. Ann Oncol 2006;17:29-34.

21. Liu C, Sun Q, Hang X, Zhong B, Wang D. Multicenter phase II study of capecitabine plus oxaliplatin as a first-line therapy in Chinese patients with advanced gastric cancer. Anticancer Drugs 2008;19:825-31.

22. Park YH, Kim BS, Ryoo BY, Yang SH. A phase II study of capecitabine plus 3-weekly oxaliplatin as first-line therapy for patients with advanced gastric cancer. Br J Cancer 2006;94:959-63.

23. Saif MW, Katirtzoglou NA, Syrigos KN. Capecitabine: An overview of the side effects and their management. Anticancer Drugs 2008;19:447-64. 